To be published in Optics Letters:

Title: $\quad$ Optical fiber lasers assisted by micro-drilled optical fiber tapers

Authors: $\quad$ Rosa Ana Pérez-Herrera,D. Pallares Aldeiturriaga,Aitor Judez,Luis Rodriguez Cobo,Manu Accepted: 20 April 19

Posted 25 April 19

Doc. ID: $\quad 361421$

(C) 2019 Optical Society of America. One print or electronic copy may be made for personal use only. Systematic reproduction and distribution, duplication of any material in this paper for a fee or for commercial purposes, or modifications of the content of this paper are prohibited. 


\title{
Optical fiber lasers assisted by micro-drilled optical fiber tapers
}

\author{
R. A. Perez-Herrera, ${ }^{1,{ }^{*}}$ D. Pallarés-Aldeiturriaga, ${ }^{2,3}$ A. Júdez,${ }^{1}$ L. \\ Rodriguez Cobo, ${ }^{3}$ M. Lopez-AMo, ${ }^{1}$ J. M. Lopez-Higuera ${ }^{2,3,4}$ \\ ${ }^{1}$ Department of Electrical Electronic and Communication Engineering and Institute of Smart Cities (ISC), Public University of Navarra, 31006 \\ Pamplona, Spain \\ ${ }^{2}$ Photonics Engineering Group, University of Cantabria, 39005 Santander, Spain \\ ${ }^{3}$ CIBER-bbn, Instituto de Salud Carlos III, 28029 Madrid, Spain \\ ${ }^{4}$ Instituto de Investigación Sanitaria Valdecilla (IDIVAL), 39005 Cantabria, Spain \\ *Corresponding author: rosa.perez@unavarra.es
}

Received XX Month XXXX; revised XX Month, XXXX; accepted XX Month XXXX; posted XX Month XXXX (Doc. ID XXXXX); published XX Month XXXX

In this letter, we present an analysis of the backscattering (BS) generated in a micro-drilled tapered fiber (MDTF) within a short-linear-cavity fiber laser. We propose and experimentally demonstrate a fiber cavity laser based on a tapered optical fiber segment that generates artificially controlled backscattering. The 50 - $\mu \mathrm{m}$-waist taper was fabricated from a standard single-mode fiber, and the taper was subsequently micro-drilled using a femtosecond laser micromachining system. The laser generation properties conferred by the micro-drilled optical fiber taper were experimentally investigated. Single-wavelength laser emission at $1562 \mathrm{~nm}$ with an output power of $10 \mathrm{dBm}$ and an optical signal-to-noise ratio (OSNR) greater than $45 \mathrm{~dB}$ is demonstrated.

http://dx.doi.org/10.1364/OL.99.099999

Artificially controlled backscattering fiber technology based on fabrication using femtosecond (fs) laser micromachining systems has developed rapidly in recent years because of the many attractive applications of these fibers across various manufacturing sectors [1-3], such as, aircraft engine turbine blades, automotive fuel filters, surgical needles, microfluidic devices, and fiber lasers [4]. This inscription technique can provide an enhancement in the inhomogeneity of the refractive index of a fiber, which is usually very weak, and consequently it offers an increase in distributed scattering [5,6]. In this way, refractive indexes can be altered and shaped to offer a number of applications for optical systems [7,8]. As an example, conventional fiber lasers require a cavity consisting of stationary mirrors or fiber loops. However, new distributed-cavity lasers [9], as well as random-fiber lasers [10], are based only on a random feedback mechanism, usually supported by backscattering, and an efficient gain than can be achieved in many different ways [10-12].
In order to provide sufficient feedback in random distributed feedback (DFB) fiber lasers, several kilometers of single-mode fiber (SMF) are usually needed. Such distances can be useful for remote sensing applications $[13,14]$, but they increase the size of laser structures and require high pump powers. Reducing the length of the distributed random mirrors has become a new research goal. Optical fiber tapers have also demonstrated their capability to suppress undesired stimulated Brillouin scattering and increase backscattering (BS) in fiber lasers [15]. Thus, DFB fiber laser operation could be dramatically improved by employing a micro-drilled tapered fiber (MDTF) in order to develop a distributed linear cavity laser.

To the best of our knowledge, this letter reports the first experimental demonstration of a fiber laser assisted by microdrilled optical fiber tapers. Here, we present an experimental analysis of fiber laser properties in terms of backscattered optical power, output power levels, and optical signal-to-noise ratio (OSNR).

Effective distributed reflection was achieved by the MDTF via multiple reflections from numerous randomly spaced spots distributed over the waist of a SMF taper. In this way, feedback strength was enhanced, and thus cavity loss was reduced and the output power threshold was lowered. A single central emission wavelength of $1562 \mathrm{~nm}$ with an output power level of $10 \mathrm{dBm}$ and an OSNR greater than $45 \mathrm{~dB}$ was obtained.

For the fabrication of the optical fiber taper, a standard optical fiber was tapered. From a nominal outer diameter of $125 \mu \mathrm{m}$, a 50$\mu \mathrm{m}$ uniform waist diameter was obtained, which corresponds to a tapering ratio of 2.5. The fabrication of the tapered fibers was executed by means of a Taper Manufacturing Station TMS-010400 (3SAE) (NorthLab Photonics, Sweden), which allows the manufacture of tapered fibers with arbitrary shapes, low losses, and excellent repeatability, as has been previously reported [16]. The transitions between the original optical fiber and the uniform 
waist of the taper were of around $5 \mathrm{~mm}$ each for a total taper length of about $30 \mathrm{~mm}$, as depicted in Fig. 1.

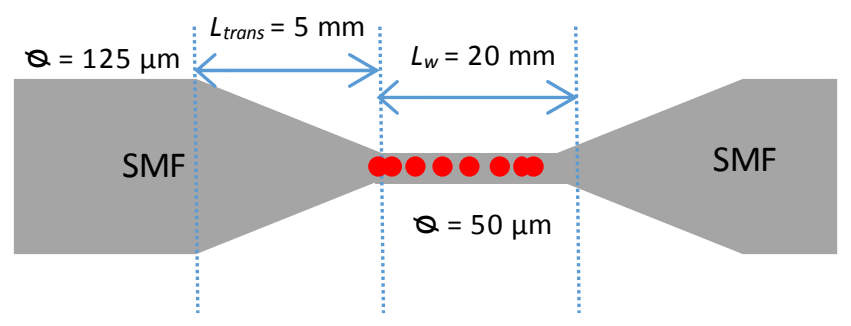

Fig. 1. Schematic illustration of the SMF microwire, to which microdrilling was applied using a femtosecond laser micromachining system.

Then, for the inscription process, a standard transversal inscription setup was selected for drilling the optical fiber taper. A commercial femtosecond fiber laser chirped pulse amplifier (FLCPA; CALMAR Laser, Inc., USA) was used to generate pulses that were focused through a NA $=0.5$ objective lens (Mitutoyo Europe GmbH; 100X Mitutoyo Plan Apo NIR Infinity Corrected Objective) to converge at the sample which was placed on a nanoresolution motorized stage (Aerotech, Inc., USA). The stage was translated in a single direction parallel to the fiber length during execution of the micro-drilling inscription. To adequately correct aberrations caused by its cylindrical geometry, the taper was placed between two standard SMFs, and all three fibers were fixed to a microscope slide. A few drops of index-matching oil were applied to the region of the taper and a cover slide was placed above this area. With this adaptive optics structure, loss of spatial resolution is avoided [17]. The femtosecond laser pulses were centered at a wavelength of $1030 \mathrm{~nm}$; the pulse duration was 370 fs and pulse energy was $0.47-0.9 \mu \mathrm{J}$; the writing speed was 0.1 $\mathrm{mm} / \mathrm{s}$. The pulse repetition rate was randomly modulated in order to inscribe the spots on the microwire with spatial periods in the range of $0-10 \mu \mathrm{m}$.

Figure 2 shows a photograph of the quasi-randomly distributed reflector along the waist of the tapered fiber. As may be observed in Fig. 2, the randomness of the inscribed structured was achieved by randomly modulating the pulse repetition rate at every update (which corresponds to $100 \mathrm{~ms}$ ). Thus, we achieved a quasi-random physically inscribed structure, with a varying envelope period of tenths of micrometers; the period was maintained for several tenths of micrometers and then randomly altered. It was not possible to inscribe randomly because the repetition rate of the femtosecond laser could not be simultaneously modulated at the same rate as the pulse inscription speed. Instead, quasi-randomly spaced spots were achieved, with random variations in spatial periods between groups of pulses, but a constant period within each envelope.

Before introducing the fabricated optical fiber taper into a linear cavity laser, the tapered fibers were characterized by means of an optical backscatter reflectometer (OBR; LUNA OBR 4600) to evaluate the backscattering over these narrowed fibers. These measurements were accomplished using the time-domain acquisition mode and with a spatial resolution of $0.1 \mathrm{~mm}$, as has been previously reported for other studies [18]. This device is used for component testing, and it allows ultra-high resolution reflectometry with a sensitivity at the level of backscattered light.

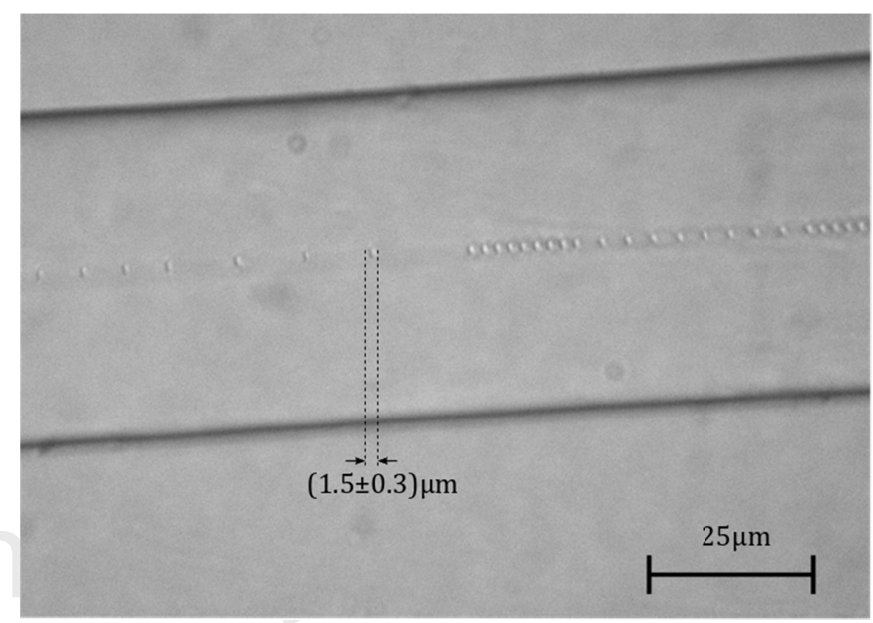

Fig. 2. Photograph of the quasi-randomly distributed reflector along the waist of the tapered fiber.
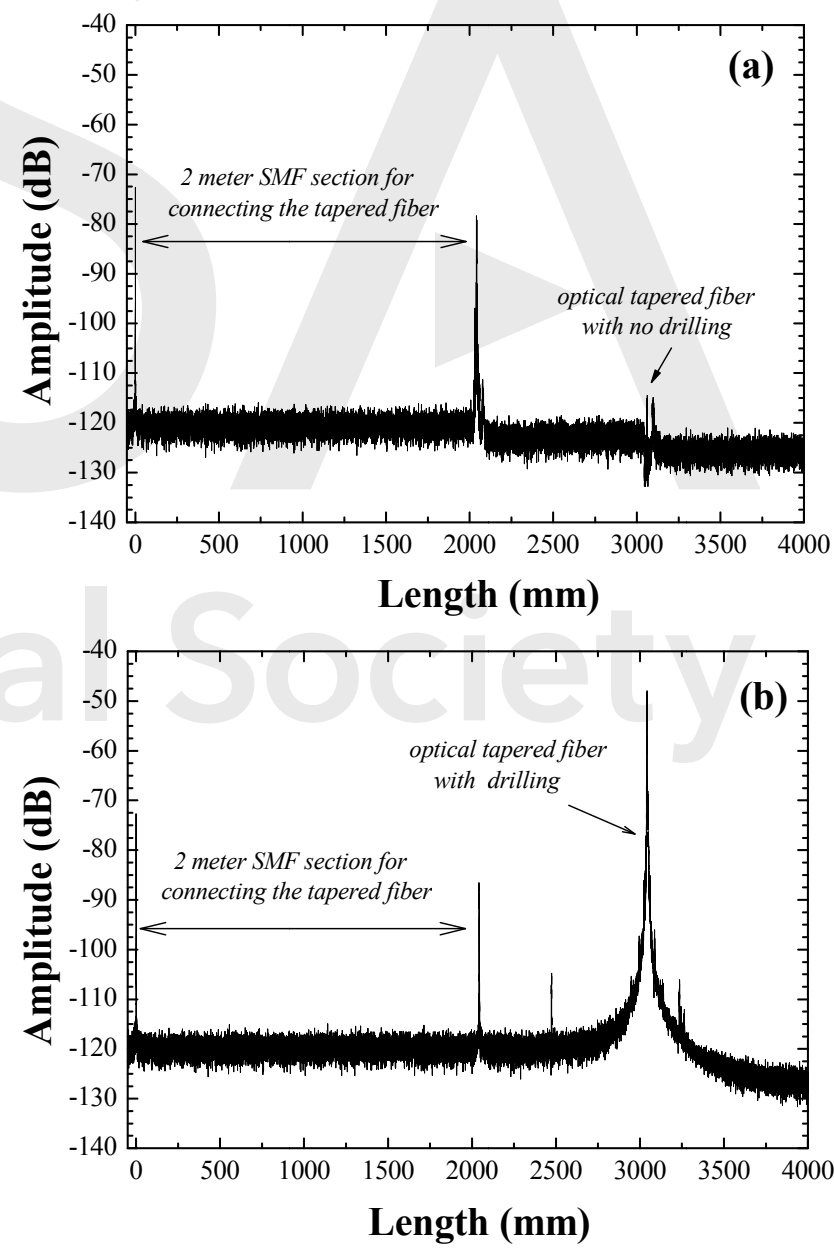
Fig. 3. Backscattered optical power as a function of fiber length for (a) the optical fiber taper with no drilling and (b) the MDTF; in each case, the fiber was located at a distance of $3 \mathrm{~m}$ from the connector of the OBR.

The OBR measurement results, depicted in Fig. 3(a) and Fig. 3(b), show the backscattered light from a 2-m SMF section as well as the SMF section that includes the taper; these two fibers were connected using pigtail splicing, as indicated by the intensity spike in the plots. As Fig. 3(a) shows, a backscattered light amplitude of about $10 \mathrm{~dB}$ over the noise floor was measured at the location of the taper, at a distance of around $3.1 \mathrm{~m}$ from the OBR connector. In contrast, when this taper was micro-drilled and connected to the OBR, a backscattered light amplitude of about $70 \mathrm{~dB}$ over the noise floor was measured, at a distance of $3 \mathrm{~m}$ from the OBR connector, as can be observed in Fig. 3(b). This increase of about $60 \mathrm{~dB}$ in amplitude is clearly due to the RBS that occurs when the tapered fiber is micro-drilled.

Next, we measured the output spectra for the linear cavity configuration, including the micro-drilled tapered fiber. A schematic diagram of the experimental setup is shown in Fig. 4. A $980 / 1550 \mathrm{~nm}$ wavelength division multiplexer (WDM) injects the pump laser centered at $976 \mathrm{~nm}$ into the linear cavity fiber laser. The gain medium, located at the common port of the WDM, consists of $4 \mathrm{~m}$ of erbium-doped fiber (EDF). The EDF was I25(980/125) (Fibercore Inc.), suitable for C amplifiers with an core composition optimized for erbium-dopes fiber amplifiers (EDFASs) in dense wavelength division multiplexing (DWDM) networks; the peak core absorption ranges from 7.7 to $9.4 \mathrm{~dB} / \mathrm{m}$ at $1531 \mathrm{~nm}$. The linear cavity of the laser ends at a fiber loop mirror (FLM) comprising an optical circulator in which ports 3 and 1 are connected. After this, the recirculating signal travels through the 1500 -nm port of the WDM to an optical coupler. At the coupler, $20 \%$ of the signal was directing into an optical spectrum analyzer (OSA) with a resolution of $0.03 \mathrm{~nm}$ to monitor the spectrum, and $80 \%$ enters the micro-drilled fiber taper. All the experimental measurements were carried out at room temperature, and no vibration isolation or temperature compensation techniques were employed.

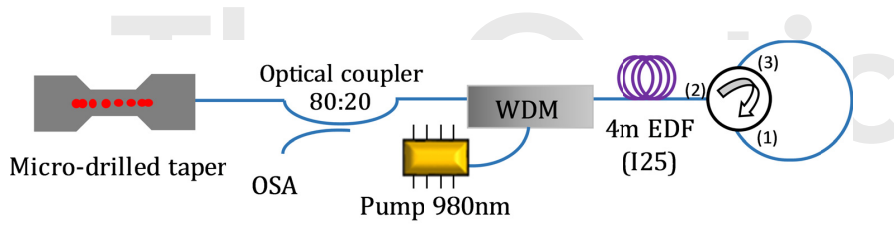

Fig. 4. Schematic diagram of the experimental linear short-cavity fiber laser setup, in which the micro-perforated fiber taper is used to reflect an amplified signal.

When the non-drilled optical fiber taper was pumped with powers in the range of $70-840 \mathrm{~mW}$, we obtained the output spectra shown in Fig. 5. As can be observed in this figure, the smallsignal gain was not sufficient for single wavelength lasing and longitudinal mode competition is observed, even when more than $800 \mathrm{~mW}$ of pump power was inserted into the cavity.

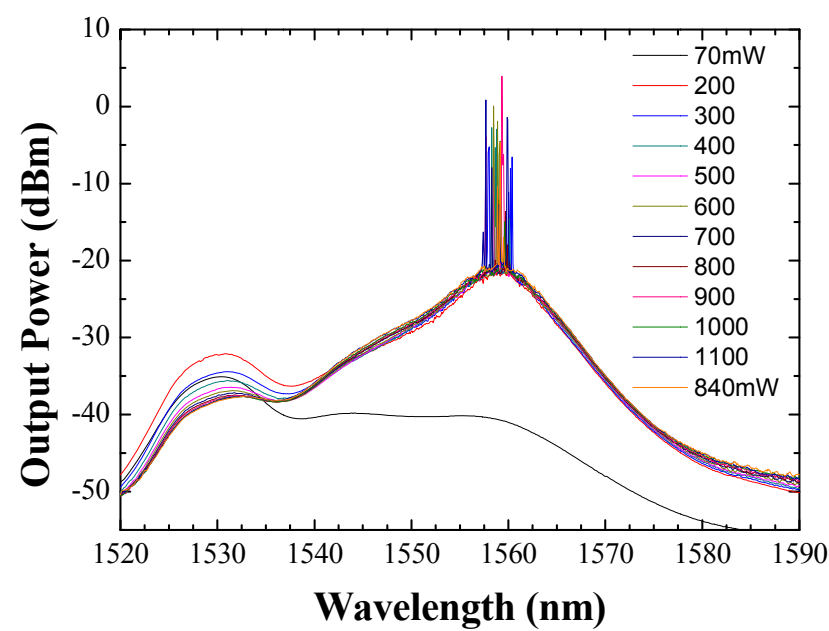

Fig. 5. Measured output spectra for the linear cavity configuration using an un-drilled optical fiber taper pumped by a 980 -nm laser at powers ranging from 70 to $840 \mathrm{~mW}$.

A very frequent event in cavities similar to the presented in this paper, that are operated above threshold, is the creation of dynamic gratings with high reflectance due to mode instabilities $[19,20]$. Such high reflective gratings may initiate spurious lasing as shown in Fig 5.

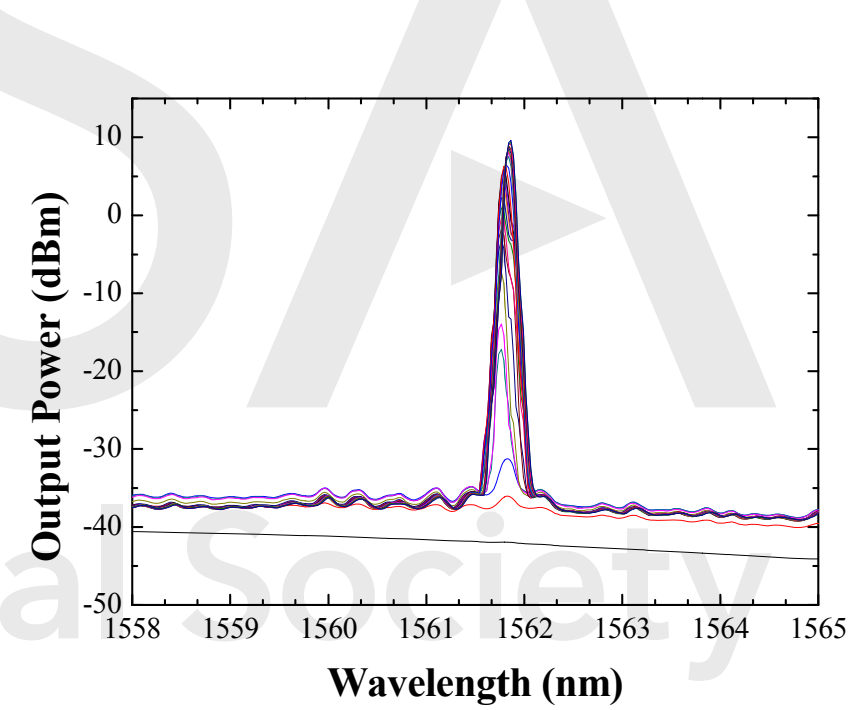

Fig. 6. Output spectra of the short-linear-cavity fiber laser with MDTF pumped by a $980-\mathrm{nm}$ laser at powers ranging from 70 to $840 \mathrm{~mW}$.

However, as Fig. 6 demonstrates, a single-wavelength laser centered at $1562 \mathrm{~nm}$ was achieved when the tapered fiber was substituted by the MDTF. The output power level obtained from this single-laser oscillation when pumped at $350 \mathrm{~mW}$ was around $10 \mathrm{dBm}$, and an OSNR of around $45 \mathrm{~dB}$ was measured. A pump power threshold of $70 \mathrm{~mW}$ was achieved, as Fig. 7 illustrates. 


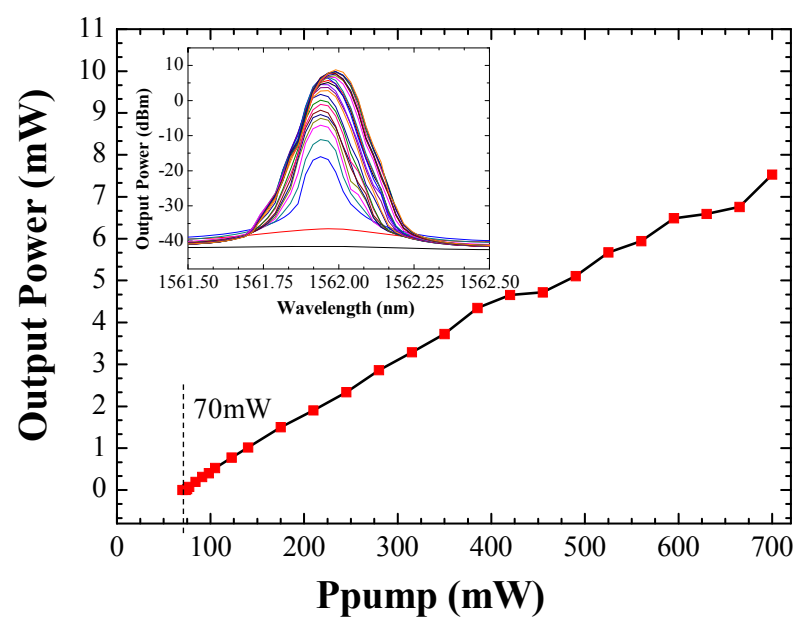

Fig. 7. Output power versus pump power for the MDTF-based fiber laser.

Finally, Fig. 8 presents a comparison between the results obtained when using a micro-drilled tapered fiber (Fig. 8(a)) or a un-drilled tapered fiber (Fig. 8(b)), both of them being pumped with $980-\mathrm{nm}$ light at $350 \mathrm{~mW}$. It is apparent that the micro-drilling has a dramatic effect on the laser generation properties.

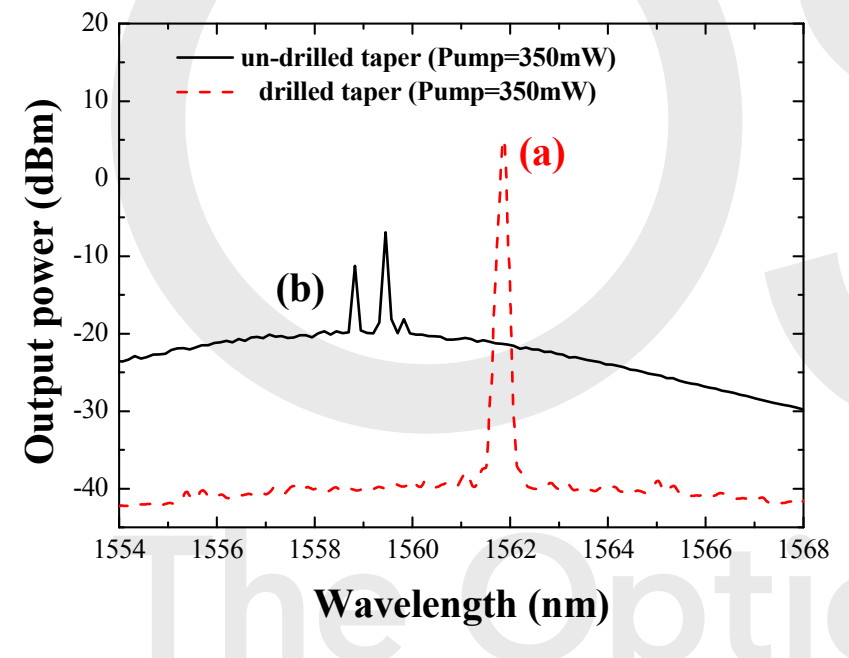

Fig. 8. Output spectra of the short-linear-cavity fiber laser pumped with $350 \mathrm{~mW}$ of light at $980 \mathrm{~nm}$ and including (a) an MDTF, or (b) a tapered fiber.

In conclusion, this letter presents, for the first time, to the best of our knowledge, an experimental analysis of the laser generation properties obtained by utilizing the effects of distributed reflections in a micro-drilled tapered fiber within a short-linearcavity fiber laser. A comparison between the light backscattered (BL) over the length of this tapered fiber, before and after it was drilled, was carried out. To this end, an optical backscatter reflectometer was employed, and it revealed an increase in the BL amplitude of about $60 \mathrm{~dB}$ for the MDTF compared to the un-drilled tapered fiber. This result is clearly due to the RBS which occurs in the micro-drilled structure. When used within the short linear cavity, the MDTF generated a single-wavelength laser centered at
$1562 \mathrm{~nm}$, with an output power of $10 \mathrm{dBm}$ and an optical signalto-noise ratio higher than $45 \mathrm{~dB}$.

Funding. MINECO (Spain) project TEC 2016-76021-C2-R; FEDER funds.

\section{References}

1. X. Wang, L. She, D. Chen, and Q. Wu, Opt. Laser Technol. 113, 6 (2019).

2. A. Ioannou, A. Theodosiou, C. Caucheteur, and K. Kalli, Opt. Lett. 42, 5198 (2017).

3. A. Theodosiou, J. Aubrecht, P. Peterka, I. Kasik, F. Todorov, O. Moravec, P. Honzatko, and K. Kalli, IEEE Photonics Technol. Lett. 31, 409 (2019).

4. H. Huang, L.-M. Yang, and J. Liu, in Proc. SPIE 8607, 86070K (2013).

5. S. Gao, L. Zhang, Y. Xu, P. Lu, L. Chen, and X. Bao, Opt. Express 24, 28353 (2016).

6. X. Wang, D. Chen, H. Li, S. H. E. Lijuan, and Q. Wu, Appl. Opt. 57, 258 (2018).

7. X. Wang, D. Chen, H. Li, and Q. Wu, Opt. Commun. 413, 224 (2018).

8. A. Consoli, E. Soria, N. Caselli, and C. López, Opt. Lett. 44, 518 (2019).

9. A. M. R. Pinto, O. Frazão, J. L. Santos, and M. Lopez-Amo, Appl. Phys. B: Lasers Opt. 99, 391 (2010).

10. S. K. Turitsyn, S. A. Babin, A. E. El-Taher, P. Harper, D. V. Churkin, S. I. Kablukov, J. D. Ania-Castãón, V. Karalekas, and E. V. Podivilov, Nat. Photonics 4, 231 (2010).

11. Z. Hu, J. Xia, Y. Liang, J. Wen, E. Miao, J. Chen, S. Wu, X. Qian, H. Jiang, and K. Xie, Opt. Express 25, 18421 (2017).

12. D. Leandro, S. Rota-Rodrigo, D. Ardanaz, and M. Lopez-Amo, J. Lightwave Technol. 33, 3591 (2015).

13. I. A. Ligato, R. A. Pérez-Herrera, M. A. Quintela, M. López-Amo, and J. M. López-Higuera, J. Lightwave Technol. 34, 4148 (2016).

14. D. Leandro, V. de Miguel-Soto, R. A. Perez-Herrera, M. B. Acha, and M. Lopez-Amo, J. Lightwave Technol. 34, 4430 (2016).

15. T. Zhu, F. Y. Chen, S. H. Huang, and X. Y. Bao, Laser Phys. Lett. 10055110 (2013).

16. A. Judez, D. Leandro, R. A. Perez-Herrera, M. Galarza, and M. Lopez-Amo, in 26th International Conference on Optical Fiber Sensors, OSA Technical Digest (Optical Society of America, 2018), paper TuE45.

17. K. Zhou, F. Shen, G. Yin, and L. Zhang, in Advanced Photonics 2016 (IPR, NOMA, Sensors, Networks, SPPCom, SOF), OSA Technical Digest (online) (Optical Society of America, 2016), paper SeW3D.1.

18. R. A. Perez-Herrera, A. Stancalie, P. Cabezudo, D. Sporea, D. Neguţ, and M. Lopez-Amo, in 26th International Conference on Optical Fiber Sensors, OSA Technical Digest (Optical Society of America, 2018), paper WF37.

19. P. Peterka, P. Koška, and J. Čtyroký, IEEE J. Sel. Topics Quantum Electron. 24, 0902608 (2018).

20. A. Yu. Tkachenko, A. D. Vladimirskaya, I. A. Lobach, and S. I. Kablukov, Opt. Lett. 43, 1558 (2018). 


\section{References}

1. X. Wang, L. She, D. Chen, and Q. Wu, "Theoretical and experimental study of artificially controlled backscattering fiber using femtosecond laser fabrication," Opt. Laser Technol. 113, 6-10 (2019).

2. A. Ioannou, A. Theodosiou, C. Caucheteur, and K. Kalli, "Direct writing of plane-by-plane tilted fiber Bragg gratings using a femtosecond laser," Opt. Lett. 42(24), 5198-5201, (2017).

3. A. Theodosiou, J. Aubrecht, P. Peterka, I. Kasik, F. Todorov, O. Moravec, P. Honzatko, and K. Kalli, "Er/Yb double-clad fiber laser with fs-laser inscribed plane-by-plane chirped FBG laser mirrors," IEEE Photonics Technol. Lett. 31(5), 409-412 (2019).

4. H. Huang, L.-M. Yang, and J. Liu, "Micro-hole drilling with femtosecond fiber laser," Proc. SPIE 8607, 86070K (2013)

5. S. Gao, L. Zhang, Y. Xu, P. Lu, L. Chen, and X. Bao, "Tapered fiber based Brillouin random fiber laser and its application for linewidth measurement," Opt. Express 24(25), 28353-28360 (2016).

6. X. Wang, D. Chen, H. Li, S. H. E. Lijuan, and Q. Wu, "Random fiber laser based on artificially controlled backscattering fibers," Appl. Opt. 57(2), 258262 (2018).

7. X. Wang, D. Chen, H. Li, and Q. Wu, "Artificially controlled backscattering in single mode fibers based on femtosecond laser fabricated reflectors," Opt. Commun. 413, 224-229 (2018).

8. A. Consoli, E. Soria, N. Caselli, and C. López, "Random lasing emission tailored by femtosecond and picosecond pulsed polymer ablation," Opt. Lett. 44(3), 518-521 (2019).

9. A. M. R. Pinto, O. Frazão, J. L. Santos, and M. Lopez-Amo, "Multiwavelength fiber laser based on a photonic crystal fiber loop mirror with cooperative Rayleigh scattering," Appl. Phys. B: Lasers Opt. 99(3), 391395 (2010).

10. S. K. Turitsyn, S. A. Babin, A. E. El-Taher, P. Harper, D. V. Churkin, S. I. Kablukov, J. D. Ania-Castãón, V. Karalekas, and E. V. Podivilov, "Random distributed feedback fibre laser," Nat. Photonics, 4(4), 231-235 (2010).

11. Z. Hu, J. Xia, Y. Liang, J. Wen, E. Miao, J. Chen, S. Wu, X. Qian, H. Jiang, and K. Xie, "Tunable random polymer fiber laser," Opt. Express, 25(15), 1842118430 (2017).

12. D. Leandro, S. Rota-Rodrigo, D. Ardanaz, and M. Lopez-Amo, "Narrowlinewidth multi-wavelength random distributed feedback laser," J. Lightwave Technol. 33(17), 3591-3596 (2015).

13. I. A. Litago, R. A. Pérez-Herrera, M. A. Quintela, M. López-Amo, and J. M. López-Higuera, "Tunable dual-wavelength random distributed feedback fiber laser with bi-directional pumping source," J. Lightwave Technol. 34(17), 4148-4153 (2016).

14. D. Leandro, V. deMiguel-Soto, R. A. Perez-Herrera, M. B. Acha, and M. López-Amo, "Random DFB fiber laser for remote $(200 \mathrm{~km})$ sensor monitoring using hybrid WDM/TDM," J. Lightwave Technol. 34(19), 4430-4436 (2016).

15. T. Zhu, F. Y. Chen, S. H. Huang, and X. Y. Bao, "An ultra-narrow linewidth fiber laser based on Rayleigh backscattering in a tapered optical fiber," Laser Phys. Lett. 10(5), 055110 (2013).

16. A. Judez, D. Leandro, R. A. Perez-Herrera, M. Galarza, and M. Lopez-Amo "High sensitivity fiber-optic liquid level sensor using biconical tapered fibers," in 26th International Conference on Optical Fiber Sensors, OSA Technical Digest (Optical Society of America, 2018), paper TuE45.

17. K. Zhou, F. Shen, G. Yin, and L. Zhang, "Optical fiber micro-devices made with femtosecond laser," in Advanced Photonics 2016 (IPR, NOMA, Sensors, Networks, SPPCom, SOF), OSA Technical Digest (online) (Optical Society of America, 2016), paper SeW3D.1.

18. R. A. Perez-Herrera, A. Stancalie, P. Cabezudo, D. Sporea, D. Neguţ, and M. Lopez-Amo, "Gamma radiation measurements using an optical fiber laser," in 26th International Conference on Optical Fiber Sensors, OSA Technical Digest (Optical Society of America, 2018), paper WF37.

19. P. Peterka, P. Koška, and J. Čtyroký, "Reflectivity of superimposed Bragg gratings induced by longitudinal mode instabilities in fiber lasers," IEEE J. Sel. Topics Quantum Electron. 24, 0902608 (2018).
20. A. Yu. Tkachenko, A. D. Vladimirskaya, I. A. Lobach, and S. I. Kablukov, "Michelson mode selector for spectral range stabilization in a self-sweeping fiber laser," Opt. Lett. 43, 1558-1561 (2018). 